\title{
Anisotropic diffusion at a melting surface studied with He-atom scattering
}

\author{
J. W. M. Frenken, ${ }^{*}$ B. J. Hinch, J. P. Toennies, and Ch. Wöll \\ Max-Planck-Institut für Strömungsforschung, Bunsenstrasse 10, D-3400 Göttingen, Federal Republic of Germany
}

(Received 3 August 1988; revised manuscript received 17 April 1989)

\begin{abstract}
Two-dimensional self-diffusion processes at surfaces can be studied on an atomic scale with quasielastic scattering of low-energy $\mathrm{He}$ atoms. The analytical strength of this new application of $\mathrm{He}$ atom scattering is demonstrated for the $\mathrm{Pb}(110)$ surface at temperatures close to the bulk melting point, $T_{m}^{\mathrm{Pb}}=600.7 \mathrm{~K}$. The width of the quasielastic-scattering energy distribution of diffusely scattered $\mathrm{He}$ atoms is a direct measure of the lateral atomic mobilities at the surface. The results show that at $T \geq \frac{3}{4} T_{m}^{\mathrm{Pb}}$ the atoms of this surface have noticeable lateral diffusivities. Above $\sim 535 \mathrm{~K}$ the surface mobilities exceed the bulk-liquid value. Measurements of the quasielastic energy broadening as a function of parallel momentum transfer provide direct information on the surface diffusion mechanisms. The results exhibit a strong directional anisotropy. The diffusion can be described in

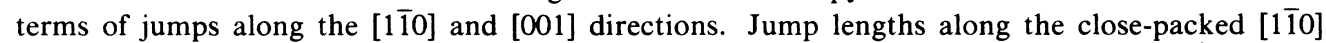
direction seem to be continuously distributed around an average jump length of $\sim 4.4 \AA$. Along [001] the diffusion proceeds in jumps over single lattice spacings.
\end{abstract}

\section{INTRODUCTION}

By now, it is well established that crystal surfaces can undergo a continuous and reversible order-disorder transition, called surface melting, at temperatures below the bulk melting point $T_{m} \cdot{ }^{1}$ Several experimental techniques have been employed to acquire detailed information on the disordering process. $^{1-8}$ As the temperature approaches $T_{m}$, the thickness of the disordered surface region diverges. The melt depth depends critically on the crystal face; the most densely packed faces remain stable up to $T_{m}$, and the most open surfaces display the strongest and earliest surface-melting effect. ${ }^{3}$

So far, the main emphasis in experimental work on surface melting has been on the loss of crystalline order at the surface. An experimental distinction between a liquidlike surface layer and a strongly disordered surface region (i.e., microcrystalline or glassy) is required in order to decide whether or not the disordered surface layers can be correctly described as a "quasiliquid."

Molecular-dynamics simulations suggest that the atoms of a melting surface have liquidlike diffusivities parallel to the surface plane. ${ }^{9,10}$ Such high mobilities cannot be measured with the conventional methods used for atomic-scale studies of surface self-diffusion, e.g., field-ion microscopy ${ }^{11}$ or the field-emission currentfluctuation technique. ${ }^{12}$ At high temperatures, selfdiffusion on the surfaces of three-dimensional solids has been investigated only with macroscopic-scale techniques, such as mass-transfer and tracer-diffusion measurements. $^{13,14}$ Atomic-scale information on surface diffusivities just below $T_{m}$ has so far only been obtained for thin methane films adsorbed on $\mathrm{MgO}$ powder, with the quasielastic scattering of thermal-energy neutrons. ${ }^{15,16}$

Here, we show that low-energy $\mathrm{He}$-atom scattering, when measured with sufficient energy resolution, can be used to probe directly the intrinsic lateral diffusion of surface atoms of a three-dimensional metal crystal exhibiting surface melting. Experimental observations are presented for $\mathrm{Pb}(110)$. Part of these have been reported before. ${ }^{4}$ At high temperatures, the elastic peak, originating from diffusely scattered $\mathrm{He}$ atoms, is observed to be broadened in energy. The results indicate that, at temperatures above $\frac{3}{4} T_{m}$, the surface atoms have anomalously high diffusivities, compared to the bulk diffusion coefficient at those temperatures. Above $\sim 535 \mathrm{~K}$, the surface selfdiffusion coefficient is larger than the diffusion constant of bulk-liquid $\mathrm{Pb}$ at $T_{m}$. The dependence of the quasielastic energy broadening, on the parallel momentum transfer, is used to obtain insight into the diffusion mechanism, distinguishing between such processes as continuous diffusion, jump diffusion, two-dimensional gaslike flight, etc. These measurements were carried out at a crystal temperature of $521 \mathrm{~K}$ and indicate that surface diffusion takes place in the form of discrete jumps. The jump length along the [001] direction (Fig. 1) seems to be restricted to single-lattice-parameter distances (4.94 $\AA$ ), whereas the jump lengths along the less corrugated, [1 $1 \overline{10}]$ direction are continuously distributed, with an average jump length of $\sim 4.4 \AA$.

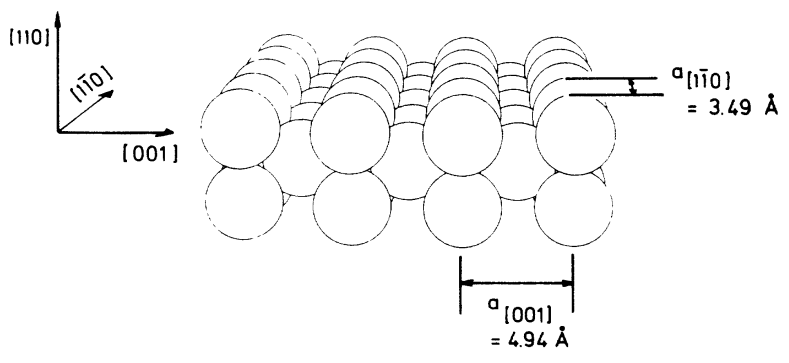

FIG. 1. Perspective view of the $\mathrm{Pb}(110)$ surface. 
The present paper is organized as follows. In the next section we present a theory for the quasielastic scattering of $\mathrm{He}$ atoms from diffusing defects on a crystal surface. In the following section the apparatus and experimental procedures are described. The experimental results are then presented and analyzed in terms of the theory. The paper closes with a discussion of our experimental findings in comparison with the available literature.

\section{THEORY OF QUASIELASTIC ATOM SCATTERING}

The principle of quasielastic scattering of low-energy atoms from laterally diffusing objects at a surface is described by the two-dimensional analogue of the theory by Van Hove, for the scattering of slow neutrons. ${ }^{17}$ When a beam of $\mathrm{He}$ atoms is elastically scattered from a surface containing a limited concentration of diffusing atoms, the energy distribution of diffusely scattered $\mathrm{He}$ atoms is, in fact, weakly inelastic. The broadening of the reflected energy distribution, with respect to the incident energy distribution, is brought about by small energy transfers, which are related to the diffusive motion of the surface atoms. The origin of these energy transfers can be explained in analogy to Doppler broadening.

It was shown by Van Hove, for the case of neutrons, and by Levi et al. ${ }^{18}$ for $\mathrm{He}$ atoms, that the quasielastic scattering cross section is related to the Fourier transform of the correlation function, $G(\mathbf{r}, t)$, between pairs of atoms. Classically, the operator $G(\mathbf{r}, t)$ is interpreted as the probability of finding an atom at the position $r$ at time $t$, given there was (will be) an atom at the origin at time $t=0$. The scattering cross section is proportional to the scattering function ${ }^{19}$

$$
S(\mathbf{k}, \omega)=\iint \exp [i(\mathbf{k} \cdot \mathbf{r}-\omega t)] G(\mathbf{r}, t) d \mathbf{r} d t,
$$

where $\hbar \mathbf{k}$ is the momentum transfer (in the following $\mathbf{k}$ will be called the momentum transfer) and $\hbar \omega$ the energy transfer in the scattering process. Departing from the convention of earlier work of our group, we will denote the momentum transfer by $\mathbf{k}$ instead of $\Delta \mathbf{k}$, since the symbol $\Delta$ will be used to designate a broadening instead of a transfer. $G(\mathbf{r}, t)$ is separable into two components:

$$
G(\mathbf{r}, t)=G_{s}(\mathbf{r}, t)+G_{d}(\mathbf{r}, t),
$$

where $G_{s}$ is the contribution to $G$ of that atom which starts at $\mathbf{r}=0, t=0$ (the self-correlation function), and $G_{d}$ is the contribution of all the other atoms (the distinctcorrelation function). Vineyard has introduced a convolution approximation, with which one can express $G_{d}$ in terms of $G_{s} .{ }^{20}$ Consequently, one finds that the scattering cross section is simply proportional to the Fourier transform of the self-correlation function, $G_{s}: 20$

$$
S(\mathbf{k}, \omega) \sim \iint \exp [i(\mathbf{k} \cdot \mathbf{r}-\omega t)] G_{s}(\mathbf{r}, t) d \mathbf{r} d t .
$$

The form of $G_{s}$ can thus be obtained directly from scattering measurements of $S(\mathbf{k}, \omega)$. Since $G_{s}$ describes the motion of individual particles, it is of primary interest in the characterization of diffusion mechanisms.

Because $G_{s}(\mathbf{r}, t)$ in fact describes both the vibrational and diffusive motion of the scatterers, it can be expressed as the convolution in space of the diffusional correlation function, $G_{s}^{D}$, with the vibrational correlation function, $G_{s}^{V}$. The Fourier transform in space and time of this convolution is equal to the energy convolution of the Fourier transforms, $S^{D}(\mathbf{k}, \omega)$ and $S^{V}(\mathbf{k}, \omega)$, of $G_{s}^{D}$ and $G_{s}^{V}$, respectively, ${ }^{21}$

$$
S(\mathbf{k}, \omega) \sim \int S^{D}\left(\mathbf{k}, \omega^{\prime}\right) S^{V}\left(\mathbf{k}, \omega-\omega^{\prime}\right) d \omega^{\prime} .
$$

The diffusive motion leads to a broadening of the energy distribution of both elastically scattered particles (elastic peak) and inelastically scattered particles (e.g., phonon-creation and -annihilation peaks). For random, continuous diffusion in two dimensions, $G_{s}^{D}$ has a Gaussian form,

$$
G_{s}^{D}(\mathbf{R}, t)=\frac{1}{4 \pi D|t|} \exp \left(-\frac{R^{2}}{4 D|t|}\right),
$$

with $\mathbf{R}$ lying in the surface plane, $R \equiv|\mathbf{R}|$, and $D$ the diffusion coefficient. The Fourier transform in Eq. (3) then leads to a Lorentzian profile of the quasielastic peak: ${ }^{17,18,20}$

$$
S^{\mathrm{qe}}(\mathbf{K}, \omega) \sim \frac{D K^{2}}{\omega^{2}+D^{2} K^{4}} .
$$

Here, $\mathbf{K}$ is the component of the momentum transfer $\mathbf{k}$ parallel to the surface, and $K \equiv|\mathbf{K}|$. Clearly, the full width at half maximum (FWHM) of this energy distribution depends on the diffusion coefficient $D$ and the parallel momentum transfer $\mathbf{K}$,

$$
\Delta E(\mathbf{K})=2 \hbar D K^{2} \text {. }
$$

We now consider the effects of a generalized discretejump diffusion mechanism in two dimensions. It has been shown by Chudley and Elliott ${ }^{21}$ that when diffusion takes place over well-defined jump vectors $\mathbf{j}$,

$$
S^{\mathrm{qe}}(\mathbf{K}, \omega) \sim \frac{f(\mathbf{K})}{\omega^{2}+f^{2}(\mathbf{K})},
$$

where

$$
\begin{aligned}
f(\mathbf{K}) & =\frac{1}{\tau} \sum_{\mathbf{j}}[1-\exp (i \mathbf{K} \cdot \mathbf{j})] P(\mathbf{j}) \\
& =\frac{1}{\tau}\left(1-\sum_{\mathbf{j}}[\cos (\mathbf{K} \cdot \mathbf{j}) P(\mathbf{j})]\right) .
\end{aligned}
$$

Here, $\tau$ is the average time between successive jumps and $P(\mathrm{j})$ is the probability for jumps over $\mathrm{j}$, which is taken to be symmetric; namely $P(-\mathrm{j})=P(\mathrm{j})$. When the possible $\mathrm{j}$ 's form a set of jumps to nearest-neighbor sites on a rectangular lattice, with lattice parameters $a_{x}$ and $a_{y}$, Eq. (9) leads to

$$
\Delta E(\mathbf{K})=\frac{2 \hbar}{\tau_{x}}\left[1-\cos \left(K_{x} a_{x}\right)\right]+\frac{2 \hbar}{\tau_{y}}\left[1-\cos \left(K_{y} a_{y}\right)\right],
$$

where $\tau_{x}$ and $\tau_{y}$ are the average times between jumps in the (positive or negative) $x$ and $y$ directions, respectively. $K_{x}$ and $K_{y}$ are the $x$ and $y$ components of the scattering 
vector $\mathbf{K}$.

When the jumps are not restricted to lattice vectors, the summation in Eq. (9) is replaced by an integral over the distribution of allowed jumps

$$
\Delta E(\mathbf{K})=\frac{2 \hbar}{\tau}\left(1-\int \cos (\mathbf{K} \cdot \mathbf{j}) P(\mathbf{j}) d \mathbf{j}\right) .
$$

Note that, at sufficiently small values of $K$, Eq. (7) is valid for all diffusion models, since information on the microscopic details of the diffusion is lost at sufficiently small $K$ values, or, equivalently, for large enough length scales. Equation (7) also implies that the specular scattering (at $\mathbf{K}=0$ ) is always purely elastic $(\Delta E=0)$.

The diffuse, elastically scattered intensity arises from the presence of defects on the surface. He-atom scattering is sensitive to defects such as adatoms, vacancies, and step edges. The latter is not expected to be as mobile as the other types of defects. ${ }^{22}$ Assuming that adatoms have a higher cross section for diffuse scattering than vacancies, ${ }^{23}$ we can determine, from our low-energy $\mathrm{He}$-atomscattering measurements, the diffusion coefficients and diffusion mechanisms for $\mathrm{Pb}$ adatoms on the $\mathrm{Pb}(110)$ surface.

\section{EXPERIMENT}

The use of $\mathrm{Pb}$ for these initial experiments has several advantages. $\mathrm{Pb}$ has a very low melting point, $T_{m}=600.7$ $K$, and at this temperature its vapor pressure is only $7 \times 10^{-7} \mathrm{~Pa}$. This allows easy control of the temperature and experiments under ultrahigh-vacuum conditions. In addition, $\mathrm{Pb}$ surfaces do not tend to contaminate, especially not at high temperatures, which makes it possible to perform long measurements on $\mathrm{Pb}$ without contamination problems.

The $\mathrm{Pb}(110)$ specimens were spark-cut from a singlecrystal ingot of $99.99 \%$ purity. An etch-polish mixture of $20 \mathrm{vol} \%$ hydrogen peroxide and $80 \mathrm{vol} \%$ acetic acid was used to remove the damaged surface region and to obtain a smooth, shiny surface. Grooves in the sides of the sample were used to clamp it in a Mo holder, which could be heated radiatively from the reverse side. The crystal temperature was monitored with a Pt-resistance thermometer and an infrared pyrometer, calibrated in situ against the bulk melting point of $\mathrm{Pb}$. The (110) surface was cleaned by cycles of Ar- or Xe-ion sputtering and annealing or by sputtering at elevated temperature. ${ }^{2}$ Surface cleanliness and crystalline order were checked with Auger-electron spectroscopy (AES) and $\mathrm{He}$ diffraction. All measurements reported here were performed under ultrahigh-vacuum conditions (base pressure of $4 \times 10^{-11}$ mbar). With the crystal at tempertures close to melting, the pressure remained in the low $-10^{-10} \mathrm{mbar}$ range. It was checked that, even after prolonged measurements of $10-30 \mathrm{~h}$ at high temperatures, contamination of the $\mathrm{Pb}(110)$ surface remained below the AES detection limit. Such long heat treatments also did not lead to reduction of the He-diffraction intensities, a particularly sensitive method for the detection of contaminants or defects.

The He-atom-scattering experiments were performed with supersonic He beams, expanding from a nozzle- skimmer configuration, as schematically shown in Fig. $2 .^{24-26}$ Unusually low beam energies, between 2.2 and $6.5 \mathrm{meV}$, were obtained by cooling the (10- $\mu \mathrm{m}$-diam) nozzle to temperatures between 10 and $31 \mathrm{~K}$. Typical $\mathrm{He}$ source pressures were around 3 bars. Scattered He atoms were detected at a fixed scattering angle of $90^{\circ}$ with respect to the incident beam, by electron-impact ionization and He-ion counting. Not shown in Fig. 2 is the extensive differential pumping, necessary to maintain the large $\mathrm{He}$ partial-pressure ratio between source and detector. Energy distributions of scattered $\mathrm{He}$ atoms were obtained by measurements of the flight time between chopper 1 and the detector. The energy resolution of the complete system, which includes the energy width of the incident $\mathrm{He}$ beam and the time resolution of the time-offlight (TOF) measurement, amounted to $\sim 80$ to $\sim 170$ $\mu \mathrm{eV}$ for beam energies between 2.2 and $6.5 \mathrm{meV} .{ }^{27}$ This was determined from measurements at room temperature or measurements of the (purely elastic) specular beam.

As the ratio of quasielastic signal to inelastic background was extremely low at high crystal temperatures, measurement times of up to $30 \mathrm{~h}$ were needed for a sufficiently precise determination of the quasielastic peak width at each angular setting. In order to raise the signal-to-background ratio, a second chopper was introduced, located between the crystal and the detector, to run in phase, to within $\pm 2.5 \mu \mathrm{s}$, with chopper $1 .^{28}$ Chopper 2 serves to select a specific time window $\Delta t$ in the TOF spectrum. This time window is much narrower than the width $\Delta T$ of the complete TOF distribution (both $\Delta t$ and $\Delta T$ are evaluated at the detector). The remaining fraction of the TOF distribution, $(\Delta T-\Delta t) / \Delta T$, is not transmitted by chopper 2 . Thus, more He pulses may be started at chopper 1 without signal overlap. The rate at which the selected part of the TOF distribution is acquired can thus be increased by a factor $\Delta T / \Delta t$. The maximum gain factor is, however, limited by the requirement that no signal be transmitted

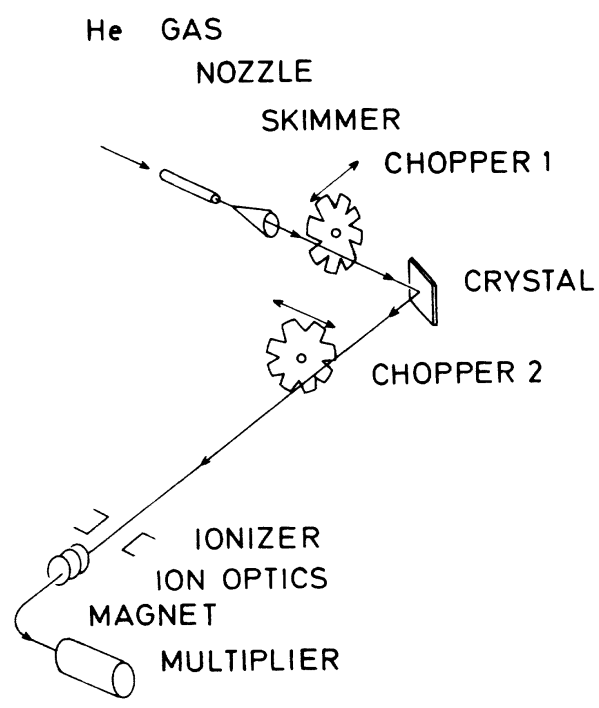

FIG. 2. Schematic representation of the atom-scattering apparatus. 
through earlier or later time windows. $\Delta T / \Delta t$ depends on the crystal-chopper and crystal-detector distances $d_{\mathrm{CC}}$ and $d_{\mathrm{CD}}: \Delta T / \Delta t=2 \mathrm{~d}_{\mathrm{CD}} / d_{\mathrm{CC}}$. For our setup the maximum gain factor amounts to $\Delta T / \Delta t=6$. In addition, chopper 2 reduces the contribution to the background signal due to $\mathrm{He}$ gas diffusing from the scattering chamber through the flight path into the detector. For the measurements reported here, the phase difference between the two choppers was selected such that the time windows were centered around the time of arrival of each quasielastic peak.

\section{RESULTS}

\section{A. Temperature dependence of diffusion}

For purposes of orientation, Fig. 3 shows a reciprocalspace diagram of the $\mathrm{Pb}(110)$ surface. The quasielastic energy distributions reported in this section were measured at the Brillouin-zone-boundary positions marked by the squares along the [001] and [110] azimuths.

Displayed in Fig. 4 is a selection of measured energy spectra of $\mathrm{He}$ atoms scattered from $\mathrm{Pb}(110)$ at crystal temperatures of 446,544 , and $551 \mathrm{~K}$, with a beam energy of $6.5 \mathrm{meV}$ and an incident angle of $37.5^{\circ}$ with respect to the surface normal, corresponding to $|\mathbf{K}|=0.64 \AA^{-1}$ along the [001] azimuth. The measurements have been corrected by subtraction of a smoothly varying inelastic background. This procedure is illustrated in Fig. 5. The dashed Gauss curves in Fig. 4 show the instrumental energy resolution of $163 \mu \mathrm{eV}$. This could be determined independently from two types of measurements, either at room temperature or at $\mathbf{K}=\mathbf{0}$ (see Sec. II). Each measured quasielastic peak is the sum of many (typically 20) shorter measurements which have been interrupted by reference measurements at $\mathbf{K}=0$. The sum of these reference measurements was used to determine the actual instrumental energy resolution during a quasielastic-

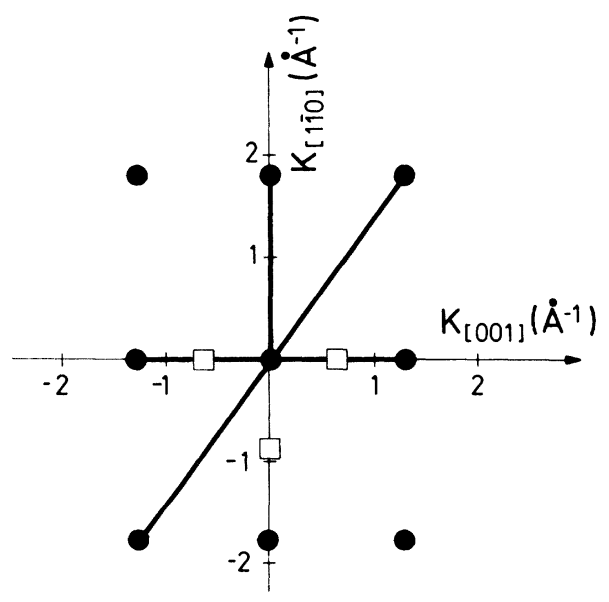

FIG. 3. Reciprocal-space diagram of the $\mathrm{Pb}(110)$ surface. The surface reciprocal lattice is indicated by the solid circles. The squares show the $\mathbf{K}$ locations for the quasielastic scattering measurements in Figs. 4, 6, and 7. The bars indicate the $|\mathbf{K}|$ ranges covered by the data in Figs. $8(a)-8(\mathrm{c})$.

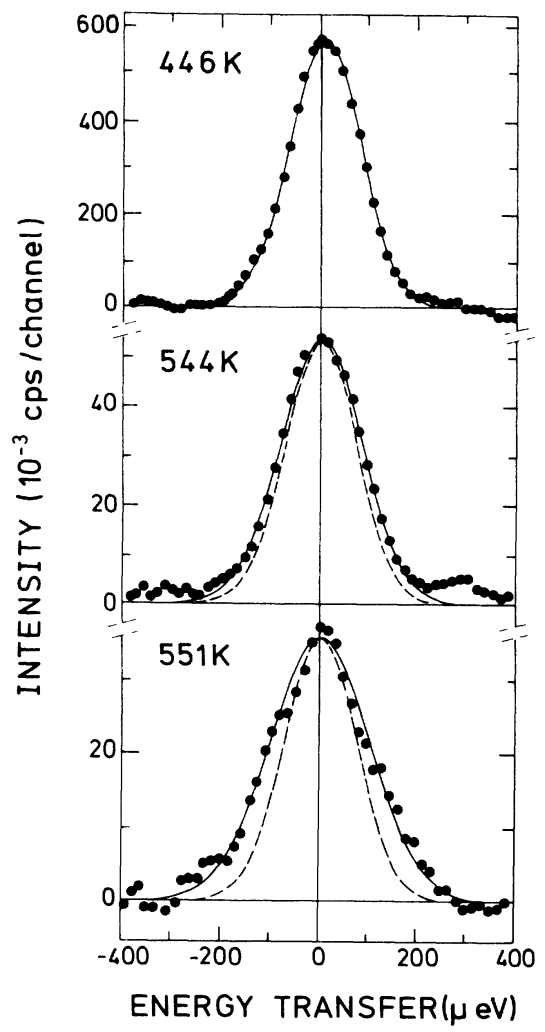

FIG. 4. Energy distributions of He atoms scattered from a $\mathrm{Pb}$ (110) surface, at three crystal temperatures, for $K=0.64 \AA-1$ along the [001] surface azimuth. The most probable beam energy is $6.5 \mathrm{meV}$. The dashed curves show the experimental resolution of $163 \mu \mathrm{eV}$. The solid curves serve to guide the eye. (cps is an abbreviation for "counts per second.")

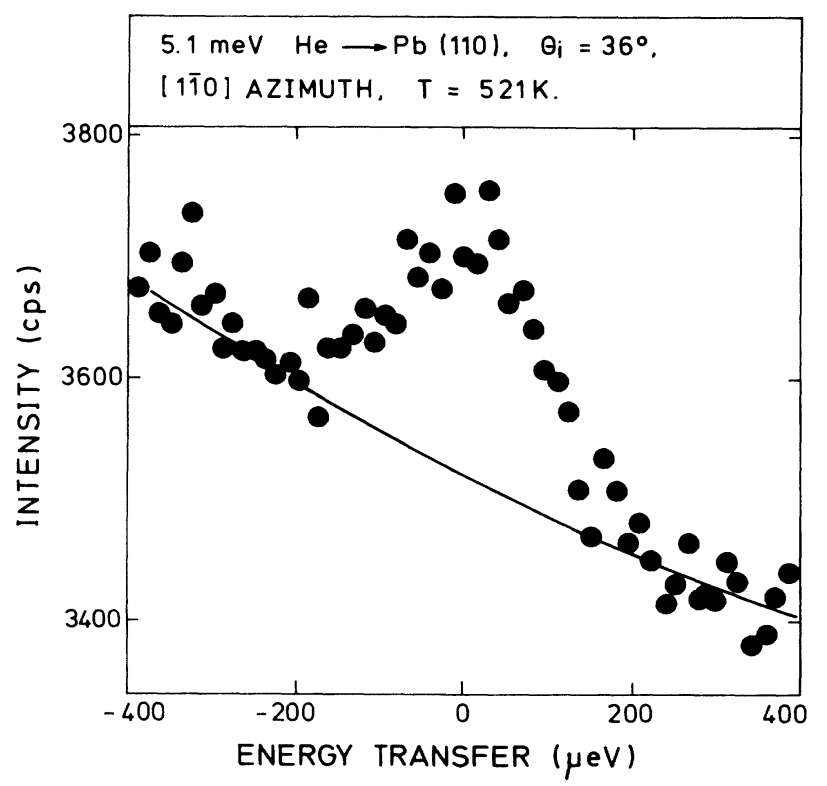

FIG. 5. Unprocessed energy spectrum, illustrating the typical signal-to-background ratio and the background subtraction. The central He energy and the angles correspond to a parallel momentum transfer of $K=0.69 \AA^{-1}$ along the [1T0] azimuth. 
scattering measurement. This eliminates the effects of possible slow variations in beam energy and beam quality.

The solid curves in Fig. 4 serve to guide the eye and are used to determine the FWHM of the measured peaks. Figure 4 clearly demonstrates the quasielastic energy broadening with increasing temperature. As is apparent from Fig. 4, the quasielastic intensity decreases substantially with temperature. This is illustrated in Fig. 6. Above $\sim 500 \mathrm{~K}$ the decrease is exceptionally strong. A similar loss of intensity is also observed for the specular peak and the diffraction peaks. This phenomenon is probably caused by the pronounced anharmonicity of the surface vibrations at these high temperatures. ${ }^{29}$ In the present experiments, at temperatures above $\sim 570 \mathrm{~K}$, the quasielastic intensities became too small to allow for a determination of its energy width.

The true energy widths $\Delta E$ were obtained after correction of the measured energy widths for the instrumental resolution. In this procedure the quasielastic energy profile was assumed to have a Lorentzian shape [Eqs. (6) and (8)], and the instrumental response was approximated by a Gaussian. Several methods are available to extract the FWHM of a Lorentzian profile from the convolution of the Lorentzian with a Gaussian instrument function of known width. ${ }^{30,31}$ In our case the subtraction of a smoothly varying inelastic background from the measured energy spectra was found to have a minor effect on the resulting peak shape, changing it from the usual Voigt shape (convolution of a Lorentzian with a Gaussian) to a profile with slightly reduced wings. In order to still reliably extract the Lorentzian widths from the measured peaks, we calibrated our width analysis by applying it to numerically constructed convolutions of our Gaussian response function with Lorentzians of various widths, superimposed on a large, smoothly varying background. Lorentzian widths obtained under the assumption that the background-subtracted profiles have an undistorted Voigt shape would have been about $5 \%$ too small.

Figure 7 shows the energy widths $\Delta E$ as a function of

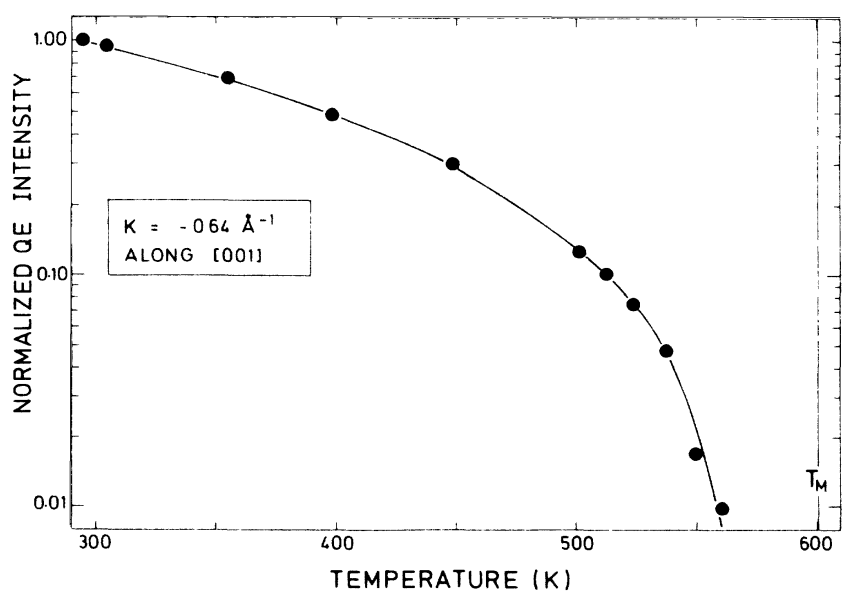

FIG. 6. Intensity of the quasielastic peak at $K=-0.64 \AA^{-1}$ as a function of temperature. The intensities have been normalized to the value at room temperature.

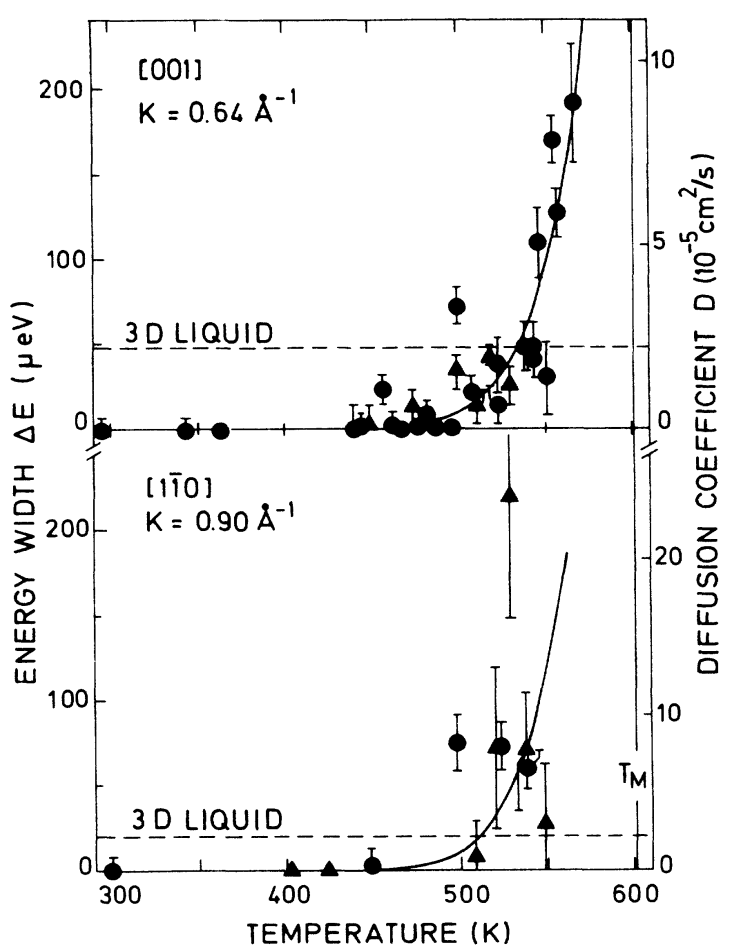

FIG. 7. Top panel: Temperature dependence of the energy width of the quasielastic peak in the energy distribution of $\mathrm{He}$ atoms scattered from $\mathrm{Pb}(110)$ with $K= \pm 0.64 \AA^{-1}$ along [001] for initial beam energies of $2.2 \mathrm{meV}$ (triangles) and $6.5 \mathrm{meV}$ (circles). The right-hand vertical axis shows the corresponding surface diffusion coefficient along the [001] direction, as described in Sec. IV B. The dashed line shows the diffusion coefficient for bulk liquid $\mathrm{Pb}$. The solid curve is discussed in the text. Bottom panel: same as top panel, for $K=-0.90 \AA^{-1}$ along the [1 10 ] surface direction.

the crystal temperature. The top panel is for $|\mathbf{K}|= \pm 0.64$ $\AA^{-1}$ along the [001] surface direction; the bottom panel is for $|\mathbf{K}|=-0.90 \AA^{-1}$ along [1 $1 \overline{1} 0$ ].

The different symbols of Fig. 7 correspond to different incident beam energies $E_{i}$. The perpendicular momentum transfer changes, between $E_{l}=2.2$ and $6.5 \mathrm{meV}$, by almost a factor 2, for a given parallel momentum transfer. Nevertheless, the observed $\Delta E$ values for the two different incident energies are, to within experimental accuracy, equal. The insensitivity to the magnitude of the perpendicular momentum transfer demonstrates that either the quasielastic $\mathrm{He}$-scattering measurements are predominantly sensitive to the lateral diffusive motion, or the diffusion coefficient in the perpendicular direction is much smaller than the lateral diffusion coefficient. In either case, the two-dimensional treatment of the quasielastic scattering given in Eqs. (5)-(11) is justified.

The measurements in Fig. 7 have been performed for $\mathbf{K}$ values at the Brillouin-zone boundary, which is far removed from the range of $\mathbf{K}$ values where $\mathrm{Eq}$. (7) is expected to be valid $(K \cong 0)$. In this $K$ region the proportionality constant linking the diffusion coefficient $D$ and the energy width $\Delta E$ is expected to be a complicated 
function of $K$, which will depend strongly on the actual diffusion mechanism (Sec. II). In the next section we show how the diffusion mechanisms along [001] and [110] for a crystal temperature of $521 \mathrm{~K}$ can be determined from measurements of $\Delta E$ as a function of $K$. These mechanisms can be used to calculate the diffusion coefficients on the right-hand vertical scale of Fig. 7. It is assumed that these diffusion mechanisms remain unchanged over the temperature range of Fig. 7. The dashed lines in Fig. 7 represent the bulk-diffusion coefficient $D_{b}=2.2 \times 10^{-5} \mathrm{~cm}^{2} \mathrm{~s}^{-1}$ of liquid $\mathrm{Pb}$ at $T_{m} \cdot{ }^{32}$ Comparison with the data shows that at the surface this value is reached already at $\sim 65 \mathrm{~K}$ below $T_{m}$ for the [001] direction and $\sim 90 \mathrm{~K}$ below $T_{m}$ for [1 $\left.1 \overline{1} 0\right]$.

The solid curve in the top panel of Fig. 7 shows the temperature dependence of the diffusion coefficient expected for the Arrhenius expression

$$
D_{s}(T)=D_{0} \exp \left(-Q_{s} / k_{B} T\right) .
$$

The data in the [001] azimuth (top panel of Fig. 7) are fitted by Eq. (12) for $Q_{s}=1.0 \mathrm{eV}$ and $D_{0}=6.2 \times 10^{4}$ $\mathrm{cm}^{2} \mathrm{~s}^{-1}, k_{B}$ being the Boltzmann constant. We estimate our choice of the activation energy $Q_{s}$ to be correct only to within $\pm 0.3 \mathrm{eV}$. The larger statistical scatter along the [110] azimuth makes it difficult to estimate $D_{0}$ and $Q_{s}$ for this direction. The solid curve in the bottom panel of Fig. 7 was calculated for $Q_{s}=1.0 \mathrm{eV}$ and $D_{0}=1.8 \times 10^{5}$ $\mathrm{cm}^{2} \mathrm{~s}^{-1}$. These results can be compared with the activation energies for self-diffusion in solid and liquid $\mathrm{Pb}$, which are 1.11 and $0.19 \mathrm{eV}$, respectively. ${ }^{32,33}$ The value of $1.0 \mathrm{eV}$ for the [001] direction on the $\mathrm{Pb}(110)$ surface is closest to that for solid $\mathrm{Pb}$. This suggests that, over the temperature range covered in Fig. 7, surface diffusion along this azimuth is noticeably affected by the presence of residual crystalline order at the surface.

At the melting point the diffusion coefficients of bulksolid and -liquid $\mathrm{Pb}$ are $4.5 \times 10^{-10}$ and $2.2 \times 10^{-5}$ $\mathrm{cm}^{2} \mathrm{~s}^{-1}$, respectively. ${ }^{32,33}$ An extrapolation of Eq. (12) with the values for $Q_{s}$ and $D_{0}$ determined for the [001] azimuth predicts a surface value of $2.5 \times 10^{-4} \mathrm{~cm}^{2} \mathrm{~s}^{-1}$ at the melting point. This finding agrees well with the result of molecular-dynamics calculations for Lennard-Jones systems; namely that, close to melting, surface diffusion coefficients are larger than bulk-liquid diffusion coefficients. 9,10

\section{B. Diffusion mechanisms}

As expressed in Sec. II, by Eqs. (5)-(11), information on the microscopic diffusion mechanism can be obtained from quasielastic atom-scattering measurements as a function of parallel momentum transfer $\mathbf{K}$. In order to obtain an atomic-scale picture of the self-diffusion on the $\mathrm{Pb}$ (110) surface, we have measured the angular dependence of the quasielastic energy width, $\Delta E$, along the two high-symmetry directions [001] and [1피, as well as the intermediate [1 $1 \overline{1} 1]$ direction (see Fig. 3). One fixed crystal temperature of $521 \mathrm{~K}$ was selected since it provided an optimal compromise between the quasielastic intensity and the diffusional energy broadening. Figure 8 displays the results, with the incident and final angles converted to

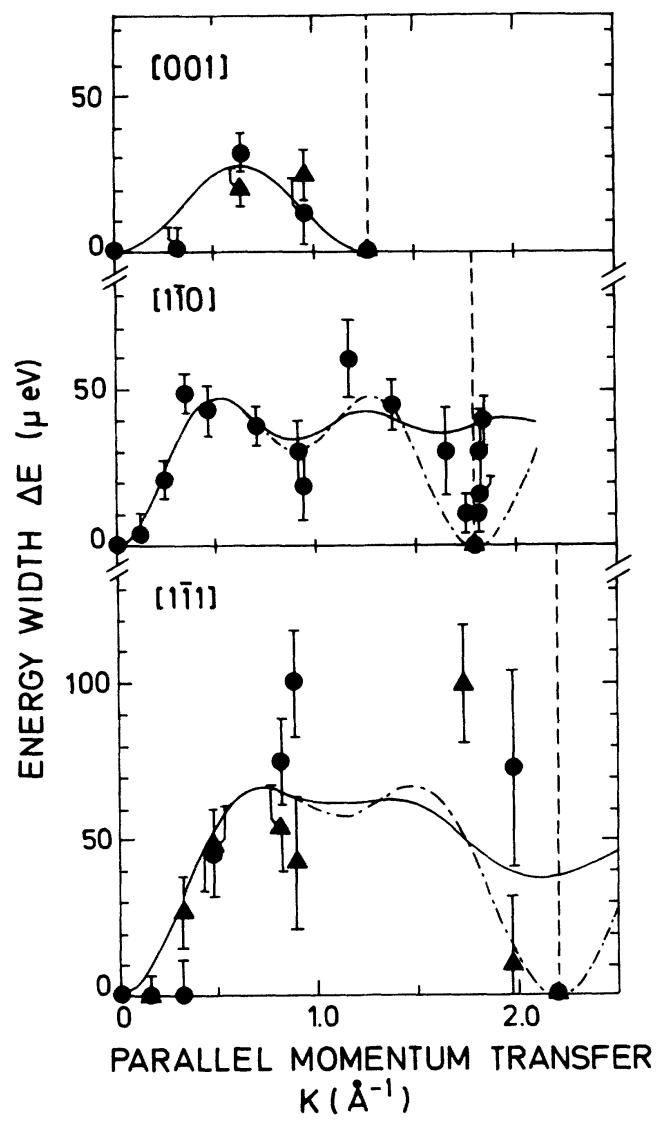

FIG. 8. $\mathbf{K}$ dependence of the energy width $\Delta E$ of the quasielastic peak, at a crystal temperature of $521 \mathrm{~K}$. Dashed vertical lines denote the reciprocal-lattice points. (a) $\mathbf{K}$ along the [001] direction. The fit, obtained for jump diffusion over single [001] distances, is discussed in the text. (b) $\mathbf{K}$ along [110]. Fits are shown for two jump-diffusion models: equally probable jumps over single and double [1 $1 \overline{1} 0]$ distances (dashed-dotted curve), and a continuous distribution of equally probable jump lengths, with a maximum jump length of $8.7 \AA$ (solid curve). Details are given in the text. (c) $\mathbf{K}$ along [111]. The dashed-dotted and solid curves have been obtained by combining the solid curve of Fig. 8(a) with the dashed-dotted and solid curves of Fig. 8(b), respectively, as described in the text.

parallel momentum transfer $\mathbf{K}$.

Several conclusions can be drawn directly from a visual inspection of Fig. 8. First, in none of the three directions is the energy width $\Delta E$ proportional to $K^{2}$ over the entire $K$ range. This shows that, at this temperature, the selfdiffusion on $\mathrm{Pb}(110)$ cannot be described as continuous, random diffusion, Eqs. (5)-(7). Second, for $K$ values smaller than $\sim 0.5 \AA^{-1}$, where the parabolic (macroscopic) description of Eq. (7) seems to be valid, the $\Delta E$ values are different along each of the three azimuths. This demonstrates that the diffusion constant depends strongly on the surface azimuthal direction. Third, for $K$ values larger than $0.5 \AA^{-1}$, the three data sets have different shapes, which indicates that the microscopic diffusion mechanisms are also different for the three directions.

More detailed conclusions about the diffusion mecha- 
nisms are reached by comparing the data in Fig. 8 with the expected $\Delta E(\mathbf{K})$ behavior for specific diffusion models. The data obtained along the [001] azimuth show one broad maximum in $\Delta E$ centered around the Brillouinzone boundary, $K=\pi / a_{[001]}$, and a minimum at the reciprocal-lattice point, $K=2 \pi / a_{[001]}=1.27 \AA^{-1}$. This shape comes closest to that expected for jump diffusion over single lattice spacings along the [001] direction [Eq. (10)]. The solid curve in Fig. 8(a) has been calculated with Eq. (10), using for the average time between successive jumps in the [001] direction $\tau_{[001]}=9.5 \times 10^{-11} \mathrm{~s}$. This value is much larger than a typical vibrational period, which is for $\mathrm{Pb}$ on the order of $1 \times 10^{-12} \mathrm{~s}^{34}$ For jump diffusion over distances $a$ with an average time $\tau$ between jumps, the diffusion coefficient can be calculated to be $D=a^{2} / 2 \tau$. Substituting the above values for $a$ and $\tau$, we find $D_{[001]}=a_{[001]}^{2} / 2 \tau_{[001]}=1.3 \times 10^{-5} \mathrm{~cm}^{2} \mathrm{~s}^{-1}$. This is an appreciable fraction of the bulk-liquid value of $2.2 \times 10^{-5} \mathrm{~cm}^{2} \mathrm{~s}^{-1}$.

The data for the [1ํㅣ azimuth, Fig. 8(b), look qualitatively different from that for [001]. The energy width increases rapidly for $K$ values up to $0.5 \AA^{-1}$, and later appears to go through a local minimum at the Brillouinzone boundary, $K=\pi / a_{[1 \overline{1} 0]}$. Then $\Delta E$ rises again and then decreases to zero at the reciprocal-lattice point $K=2 \pi / a_{[1 \overline{1} 0]}=1.80 \AA^{-1}$. Beyond this point it increases sharply to return to the value of $\sim 40 \mu \mathrm{eV}$. This swinging behavior was carefully checked by the large number of points in this region. The scattering intensities suggest that the measurements around $K=1.80 \AA^{-1}$ are dominated by a purely elastic diffraction contribution, from the $\mathrm{Pb}(110)$ substrate, which is not yet completely disordered at $521 \mathrm{~K}$. We are thus forced to ignore the few data points around $1.80 \AA^{-1}$. We then see that the quasielastic signal for this azimuth, from individual diffusing atoms on the surface, does not exhibit any other local minima in the energy width. This means that diffusion models which describe the diffusion in terms of instantaneous jumps of length $a_{[1 \overline{1} 0]}$ or integer multiples thereof cannot provide an appropriate fit to these data. This is illustrated by the dashed-dotted curve in Fig. 8(b), which has been obtained for a jump-diffusion model with equally probable jump lengths of $a_{[1 \overline{1} 0]}$ and $2 a_{[1 \overline{1} 0]}$, and an average time between successive jumps of $4.3 \times 10^{-11} \mathrm{~s}$. For this diffusion model the diffusion coefficient is given by $D=5 a^{2} / 4 \tau$. We thus find $D_{[1 \overline{1} 0]}=5 a_{[1 \overline{1} 0]}^{2} / 4 \tau_{[1 \overline{1} 0]}$ $=3.5 \times 10^{-5} \mathrm{~cm}^{2} \mathrm{~s}^{-1}$. This model fits the data in Fig. 8 (b) only up to $\sim 1.5 \AA$ and then predicts a minimum in $\Delta E$ for $K=1.80 \AA^{-1}$ which should have the same shape as the minimum around $K=0 \AA^{-1}$, in contrast to the measurements. The fact that this single- and doublejump model fits the data up to $\sim 1.5 \AA^{-1}$, including the local minimum at $0.9 \AA^{-1}$, indicates that both the diffusion coefficient and the average jump length are already approximately described by this model. The simplest alternative model, which does not lead to a distinct periodicity of $\Delta E$ in reciprocal space, allows a continuous distribution of equally probable jump lengths between zero and a maximum jump length $a_{\max }$. From the average jump length for the single- and double-jump model of
$1.5 a_{[1 \overline{10}]}$, we estimate the maximum jump length to be $a_{\max } \approx 3 a_{[1 \overline{1} 0]} \approx 10 \AA$. Fitting the continuous-distribution model [Eq. (11)] to the data leads to $a_{\max }=8.7 \AA$ and $\tau_{[1 \overline{1} 0]}=3.4 \times 10^{-11} \mathrm{~s}$, corresponding to $D_{[1 \overline{1} 0]}=a_{\max }^{2} /$ $6 \tau_{[1 \overline{1} 0]}=3.8 \times 10^{-5} \mathrm{~cm}^{2} \mathrm{~s}^{-1}$. The solid curve in Fig. $8(\mathrm{~b})$ is the resulting best fit.

Knowing the jump mechanisms and jump times along the two high-symmetry directions on the (110) surface, and assuming the jumps in these two directions to take place independently, as was implicitly done in Eq. (10), one can calculate the expected diffusion coefficient and $\Delta E(\mathbf{K})$ dependence for any intermediate direction by an appropriate linear combination of the diffusion coefficients and $\Delta E(K)$ curves along [001] and [110]. This is nicely confirmed by the data in Fig. 8(c) for the [111] azimuth, which makes an angle of $54.7^{\circ}$ with the [001] direction. The two curves in Fig. 8(c) were obtained by combining the fits in Figs. 8(a) and 8(b), according to

$$
\begin{aligned}
\Delta E_{[1 \overline{1} 1]}(K)= & \Delta E_{[001]}\left(K \cos \left(54.7^{\circ}\right)\right) \\
& +\Delta E_{[1 \overline{1} 0]}\left(K \sin \left(54.7^{\circ}\right)\right) .
\end{aligned}
$$

As for the [1 $1 \overline{1} 0]$ direction, the energy distributions along the [111] azimuth are not broadened at the reciprocallattice point $\left(2.20 \AA^{-1}\right)$, due to a dominating diffraction contribution from the substrate. The other energy widths in Fig. 8(c) are described well by the solid curve. Note that for this fit to the [1 111 ] data no additional fitting parameters have been used. The diffusion coefficient along [1111] amounts to

$$
\begin{aligned}
D_{[1 \overline{1} 1]} & =\cos ^{2}\left(54.7^{\circ}\right) D_{[001]}+\sin ^{2}\left(54.7^{\circ}\right) D_{[1 \overline{1} 0]} \\
& =2.9 \times 10^{-5} \mathrm{~cm}^{2} \mathrm{~s}^{-1} .
\end{aligned}
$$

\section{DISCUSSION}

In summary, the quasielastic He-atom-scattering measurements of diffusion at the $\mathrm{Pb}(110)$ surface, at $521 \mathrm{~K}$, are consistent with a diffusion model which comprises jumps along the [001] direction over single lattice spacings and jumps along [1 $1 \overline{1} 0]$ with a continuous distribution of jump lengths from 0 to $\sim 8.7 \AA$. Jump frequencies as well as diffusion coefficients are different for the two directions. The temperature dependence of the diffusion coefficient, Fig. 7, can be described by an Arrhenius behavior with an activation energy of $\sim 1.0$ $\mathrm{eV}$. Above $\sim 535 \mathrm{~K}$ all azimuths on the $\mathrm{Pb}(110)$ surface exhibit a diffusion coefficient exceeding the value for bulk-liquid $\mathrm{Pb}$.

Our results for the surface of a three-dimensional solid show some similarities to recent quasielastic neutronscattering results by Bienfait et al. for thin adsorbed methane films on $\mathrm{MgO}$ powder. ${ }^{15,16}$ The $\mathrm{MgO}$ particles could be shown to expose mainly (001) surfaces, on which the methane overlayers grow epitaxially. Although the (001) surfaces were, of course, randomly oriented in these experiments, the $\Delta E(\mathbf{K})$ data provided evidence for jump diffusion over single lattice spacings on a square lattice. 
Also in this study, the derived surface self-diffusion coefficients for the methane films, close to melting, were found to exceed the bulk-liquid value.

In order to explain the high-temperature behavior of the mass-transport diffusion coefficients, found on various metal surfaces, Bonzel has proposed that a nonlocalized diffusion process dominates at high temperatures. ${ }^{13}$ In this process the adatoms could diffuse by a twodimensional gaslike flight. Neither the values of the diffusion coefficients found here, nor the diffusion mechanisms extracted from our $\Delta E(\mathbf{K})$ data, support such a process to be active on $\mathrm{Pb}(110)$. Also, the large diffusion anisotropy at $521 \mathrm{~K}$ is a strong indication against a gaslike diffusive state.

Since for the $\mathrm{He}$ atoms the diffusing $\mathrm{Pb}$ atoms are, in the language of neutron scattering, "coherent" scatterers, quasielastic He-scattering measurements do not permit us to distinguish between diffusion mechanisms, in which a single adatom jumps from one site to another, and exchange mechanisms, in which the adatom changes site with a substrate atom, which, in turn, is displaced to a new adatom position. In particular, the jump diffusion across the close-packed [1힐 rows could take place in this way. In fact, at low temperatures this type of behavior has been observed experimentally for self-diffusion on the $W(221)$ surface, with the field-emission currentfluctuation technique. ${ }^{12}$

The anisotropy found here for the diffusion coefficient and the diffusion mechanism along [001] and [110] is apparently related to the anisotropic structure of the $\mathrm{Pb}$ (110) surface. As shown in Fig. 1, the (110) surface of a fcc crystal consists of close-packed [1 $1 \overline{1} 0]$ rows, separated by one lattice parameter. The corrugation in the [001] direction, perpendicular to the rows, is substantial,

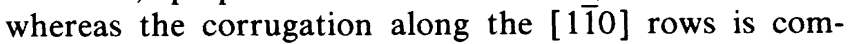
paratively weak. The activation energy for adatom diffusion might therefore be expected to be larger for the [001] direction than for the [110] direction. The statistics of the data in Fig. 7 is not good enough to directly compare the activation energies for both directions. Nevertheless, the difference in diffusion coefficients suggests that the diffusion along [001] is more difficult than along

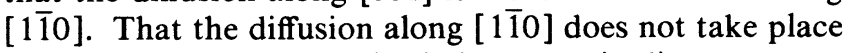
in jumps of single or multiple interatomic distances may seem surprising. However, recent low-energy electrondiffraction observations by Prince et al. have revealed that the lattice order at the $\mathrm{Pb}(110)$ surface degrades anisotropically with temperature. ${ }^{7}$ AT $521 \mathrm{~K}$ the order along the [001] direction is still almost complete, while the [1이 direction already exhibits a large degree of disorder.

Finally, we notice that the surface diffusion coefficients in Fig. 7 correlate well with the results of a recent ionscattering study from $\mathrm{Pb}(110){ }^{2}$ In this work the $\mathrm{Pb}(110)$ surface was shown to become increasingly disordered at temperatures above $\sim 450 \mathrm{~K}$. Up to $\sim 580 \mathrm{~K}$ a transition region about 10 monolayers thick forms, over which the order is gradually lost with distance from the underlying crystal to the surface. Above this temperature, this region of transition moves into the bulk, leaving a surface which looks fully disordered in the ion-scattering measurements.

The temperatures at which the anomalously strong surface diffusion is measured, in the present investigation, fall in the temperature range where the transition layer is formed. This implies that residual crystalline order is expected to play a role in the observed diffusion. The diffusion mechanism along [001] and the high value of the estimated activation energy as well as the pronounced anisotropy in the surface diffusion constant clearly demonstrate the effects of the residual crystalline order at the $\mathrm{Pb}(110)$ surface at these temperatures.

\section{CONCLUSIONS}

We have shown that quasielastic He-atom scattering can be used to obtain valuable information about lateral diffusion processes at surfaces. Diffusion coefficients as well as diffusion mechanisms can be extracted from measurements of the quasielastic energy width as a function of parallel momentum transfer. This new technique can be used to investigate self-diffusion and diffusion of adsorbates on surfaces.

The results presented here for self-diffusion at a melting $\mathrm{Pb}(110)$ surface demonstrate that the quasiliquid surface layer combines liquidlike behavior (high diffusion coefficients) with latticelike properties (lattice diffusion, azimuthal anisotropy).

At present, the energy resolution which can be reached in $\mathrm{He}$-atom-scattering experiments (typically $150 \mu \mathrm{eV}$ ) is such, that diffusion studies with this technique are only feasible for systems which feature extremely high (liquidlike) mobilities parallel to the surface plane. So far, no quasielastic energy broadening has been observed yet for an adsorbate system. Further efforts are necessary in order to improve the energy resolution of $\mathrm{He}$-atom scattering to such an extent that measurements become possible for systems with less extreme diffusivities.

\section{ACKNOWLEDGMENTS}

We thank A. J. Riemersma and A. C. Moleman of the University of Amsterdam and B. Pluis of the FOMInstitute for Atomic and Molecular Physics, (Amsterdam, The Netherlands), for the preparation of our $\mathrm{Pb}$ specimen. We thank $H$. Schief for assistance in the running of the experimental apparatus and also $\mathbf{H}$. Wuttke and J. Engelke for further technical assistance. Two of the authors (J.W.M.F. and B.J.H.) thank the Alexander von Humboldt-Stiftung (Bonn, Germany) for financial support.

\footnotetext{
*Present address: FOM-Institute for Atomic and Molecular Physics, Kruislaan 407, NL-1098 SJ Amsterdam, The Netherlands.

${ }^{1}$ J. F. van der Veen, B. Pluis, and A. W. Denier van der Gon, in Chemistry and Physics of Solid Surfaces, edited by R. Van-
}

selow and R. F. Howe (Springer, Berlin, 1988), Vol. VII, p. 455, and references therein; J. F. van der Veen and J. W. M. Frenken, Surf. Sci. 178, 382 (1986).

${ }^{2}$ J. W. M. Frenken, P. M. J. Marée, and J. F. van der Veen, Phys. Rev. B 34, 7506 (1986). 
${ }^{3}$ B. Pluis, A. W. Denier van der Gon, J. W. M. Frenken, and J. F. van der Veen, Phys. Rev. Lett. 59, 2678 (1987).

${ }^{4}$ J. W. M. Frenken, J. P. Toennies, and Ch. Wöll, Phys. Rev. Lett. 60, 1727 (1988).

${ }^{5}$ D.-M. Zhu and J. G. Dash, Phys. Rev. Lett. 57, 2959 (1986).

${ }^{6}$ J. Krim, J. P. Coulomb, and J. Bouzidi, Phys. Rev. Lett. 58, 583 (1987).

${ }^{7}$ K. C. Prince, U. Breuer, and H. P. Bonzel, Phys. Rev. Lett. 60, 1146 (1988).

${ }^{8}$ P. H. Fuoss, L. J. Norton, and S. Brennan, Phys. Rev. Lett. 60, 2046 (1988).

${ }^{9}$ V. Rosato, G. Cicotti, and V. Pontikis, Phys. Rev. B 33, 1860 (1986).

10J. Q. Broughton and G. H. Gilmer, J. Chem. Phys. 79, 5119 (1983).

${ }^{11}$ D. W. Bassett, in Surface Mobilities on Solid Materials, edited by Vu Thien Binh (Plenum, New York, 1983), pp. 63 and 83 .

${ }^{12}$ M. Tringides and R. Gomer, J. Chem. Phys. 84, 4049 (1986).

${ }^{13}$ H. P. Bonzel, in Surface Mobilities on Solid Materials, edited by Vu Thien Binh (Plenum, New York, 1983), p. 195, and references therein.

${ }^{14}$ G. E. Rhead, Surf. Sci. 47, 207 (1975).

${ }^{15}$ M. Bienfait, Europhys. Lett. 4, 79 (1987).

${ }^{16}$ M. Bienfait, J. P. Coulomb, and J. P. Palmari, Surf. Sci. 182, 557 (1987).

${ }^{17}$ L. Van Hove, Phys. Rev. 95, 249 (1954).

${ }^{18}$ A. C. Levi, R. Spadacini, and G. E. Tommei, Surf. Sci. 121, 504 (1982).

${ }^{19}$ Details of the interaction between the scattering particles (neutrons, He atoms) and the objects from which they scatter (nuclei, surfaces) do not enter our description of the quasielastic scattering. This interaction gives rise to a factor in the scattering amplitude which has already been divided out in Eq. (1).

${ }^{20}$ G. H. Vineyard, Phys. Rev. 110, 999 (1958). The proportionality factor between the left- and right-hand sides of Eq.
(3) depends on $\mathbf{k}$, as a result of simultaneous correlations in atomic positions of distinct particles. Here, we have omitted this factor, for convenience, since we only study the width of the energy $(\omega)$ profile as a function of $\mathbf{k}$, and not its amplitude.

${ }^{21}$ C. T. Chudley and R. J. Elliott, Proc. Phys. Soc. London 77, 353 (1961).

${ }^{22}$ A. Lahee, J. R. Manson, J. P. Toennies, and Ch. Wöll, Phys. Rev. Lett. 57, 471 (1986).

${ }^{23}$ E. Zaremba, Surf. Sci. 151, 91 (1985).

${ }^{24} \mathrm{G}$. Lilienkamp and J. P. Toennies, J. Chem. Phys. 78, 5210 (1983).

${ }^{25}$ D. M. Smilgies and J. P. Toennies, Rev. Sci. Instrum. 59, 2185 (1988).

26J. P. Toennies, in Surface Phonons, edited by W. Kress (Springer, Berlin, in press).

${ }^{27} \mathrm{G}$. Brusdeylins (private communication).

${ }^{28}$ H. H. Sawin, D. D. Wilkinson, W. M. Chan, S. Smiriga, and R. P. Merrill, J. Vac. Sci. Technol. 14, 1205 (1977).

${ }^{29}$ G. Armand, D. Gorse, J. Lapujoulade, and J. R. Manson, Europhys. Lett. 3, 1113 (1987).

${ }^{30} \mathrm{G}$. K. Weitheim, M. A. Butler, K. W. West, and D. N. E. Buchanan, Rev. Sci. Instrum. 45, 1369 (1974).

${ }^{31}$ In a preliminary report of the data in Fig. 7, the Lorentzian profile of the quasielastic peak has been approximated by a Gaussian (Ref. 4). In the deconvolution of the experimental energy distributions, this has led to large systematic errors in the determined quasielastic peak widths, of a factor 2-3. This accounts for the differences in energy widths and activation energies between the present work and Ref. 4 . The qualitative conclusions of Ref. 4 remain unchanged.

${ }^{32}$ N. H. Nachtrieb, Ber. Bunsenges. 80, 678 (1976).

${ }^{33}$ J. W. Miller, Phys. Rev. 181, 1095 (1969).

${ }^{34}$ The estimate of the vibrational period of $\mathrm{Pb}$ atoms of $1 \times 10^{-12} \mathrm{~s}$ is based on the typical phonon energies of $\mathrm{Pb}$, which are in the order of several milli-electron-volts. 